\title{
Determination of longitudinal bunch shape by means of coherent Smith-Purcell radiation
}

\author{
G. Doucas \\ Sub-Department of Particle Physics, University of Oxford, Oxford OX1 3RH, United Kingdom \\ M. F. Kimmitt \\ Physics Centre, University of Essex, Colchester CO4 3SQ, United Kingdom
}

A. Doria, G.P. Gallerano, E. Giovenale, and G. Messina

ENEA, Divisione Fisica Applicata, P.O. Box 65, 00044 Frascati, Italy

H. L. Andrews and J.H. Brownell

Department of Physics \& Astronomy, Dartmouth College, Hanover, New Hampshire 03755-3528

(Received 11 May 2002; published 24 July 2002)

\begin{abstract}
Coherent enhancement of the Smith-Purcell radiation produced from the interaction of a $1.8 \mathrm{MeV}$ electron beam with a grating has been observed. The emitted radiation has been measured at angles in the $40^{\circ}$ to $120^{\circ}$ range, which correspond to wavelengths from 0.65 to $4 \mathrm{~mm}$, approximately. The radiated power was $320 \mathrm{~mW}$ at $90^{\circ}$. Its angular distribution agrees well with the description of the process in terms of induced surface currents and has been used to infer the longitudinal profile of the electron bunch. It is concluded that the bunch has an approximately triangular profile, with $85 \%$ of the bunch particles contained within 14 ps. The possibilities of the technique as a bunch-shape diagnostic tool are also discussed.
\end{abstract}

DOI: 10.1103/PhysRevSTAB.5.072802

PACS numbers: 41.60.-m, 41.75.Ht, 29.27.Fh

\section{INTRODUCTION}

Noninvasive beam diagnostic techniques are likely to play an important role in the next generation of highenergy electron accelerators, whether these are used for particle physics ("Linear Collider") or as sources for x-ray free electron lasers (FELs). One possible method of obtaining information about certain properties of the beam, such as longitudinal profile of the bunch, is by observing the radiation emitted through the interaction of the beam with a medium. The oldest of these "optical" techniques is based on transition radiation (TR), produced by the passage of the bunch through a very thin foil. Strictly speaking, this is an invasive technique and a possible alternative is the diffraction radiation (DR), which can be considered as a special case of TR in which the foil has an aperture through which the beam passes. Both TR [1-3] and DR [4-7] have been used, or have been proposed, as beam diagnostic devices. A very recent addition to the armory of noninvasive techniques is the measurement of bunch duration and shape by means of the detection of the electric field of the relativistic electron as it passes close to a crystal of ZnTe [8].

Another process that is nonintercepting and causes minimal disruption to the electron beam is the so-called SmithPurcell radiation (SPR), which is the radiation produced by the interaction of the electron with a periodic structure, such as a metallic grating. This radiation has some interesting and, potentially, advantageous features: the emitted intensity is proportional to the number of periods of the grating, hence it is strong compared to, say, diffraction radiation. The wavelength region can be selected through an appropriate choice of grating period and the observed wavelength depends on the angle of observation; hence, almost all the emitted wavelengths can be collected by changing this angle. Through a suitable choice of the blaze angle of the grating it is possible to direct the radiation pattern away from the direction of the electron beam, allowing easy observation. A detailed comparison between SPR and other radiative processes is beyond the scope of the present paper, but we note that although some of the above features occur in other processes as well, SPR is the only one that combines all of them together. This combination is also suitable for a high-resolution beam position sensor for ultrarelativistic electron beams, as mentioned recently [9]. Here, we report on the results of recent experiments carried out at the ENEA facility at Frascati, Rome, and consider the potential of coherent SPR as a pulse-shape diagnostic tool for picosecond electron bunches.

\section{PHYSICAL PRINCIPLES}

\section{A. One-electron case}

When an electron passes close to the surface of a metallic grating, radiation is emitted because of the interaction of the particle with the periodic structure. The wavelength $\lambda$ of the emitted radiation depends on the period $l$ of the grating, the speed of the electron, expressed in terms of $\beta$, and also on the angle of observation $\theta$ relative to the electron's velocity, i.e.,

$$
\lambda=\frac{l}{n}\left(\frac{1}{\beta}-\cos \theta\right),
$$


where $n$ is the order of the radiation. This equation was first reported in 1953 by Smith and Purcell [10] and is now well established experimentally. It is thus possible to select a wavelength by varying, for example, the angle of observation $\theta$. The spectrum condenses to discreet orders and this is another distinct feature of SPR, as it is possible to select harmonics higher than 1 . The calculation of the emitted power and its angular distribution requires some kind of "model" for the emission process. One approach to this problem is to ascribe the origin of this radiation to surface currents induced on the grating by the passing electron and "accelerated" by the periodic profile of the grating [11,12]. The details of this calculation are omitted here but the conclusions can be summarized by noting that the angular and frequency distribution of the intensity emitted by a single electron passing at a height $x_{0}$ over a single period $l$ of the grating is given by

$$
\frac{d^{2} I}{d \omega d \Omega}=\frac{e^{2} \omega^{2} l^{2}}{4 \pi^{2} c^{3}} R^{2} \exp \left[\frac{-2 x_{0}}{\lambda_{e}}\right] .
$$

The quantity $\lambda_{e}$ that appears in (2) is the so-called "evanescent wavelength" and is defined in terms of the emitted wavelength $\lambda$, the observation angles $\theta$ and $\phi$, and the relativistic parameters of the beam by the expression:

$$
\lambda_{e}=\lambda \frac{\beta \gamma}{2 \pi \sqrt{1+\beta^{2} \gamma^{2} \sin ^{2} \theta \sin ^{2} \phi}} .
$$

The term $R^{2}$ is the magnitude of a complex vector [see Eq. (10) of Ref. [11]] and is a complicated expression that depends on the details of the grating profile and on the wavelength of the radiation. Although an analytic form can be written down for the case of a profile consisting of two straight facets only, it is best to evaluate $R^{2}$ numerically. Typically, for the experimental conditions of the present paper, $R^{2}$ is of the order $10^{-2}$ to $10^{-1}$.

For a grating with $N$ periods and, hence, overall length $Z=N l$, the situation is analogous to that of a linear array of equal oscillators. Equation (1) defines the direction where a particular wavelength component of all $N$ wavelets arrives in phase at the detector. The total spectral intensity varies as $N^{2}$, but the resolution is limited to $d \omega / \omega \cong$ $1 / n N$, so that the radiated intensity, for one electron, is $N$ times that of a single facet, i.e., proportional to the grating length $Z$ :

$$
\left(\frac{d I}{d \Omega}\right)_{1}=\frac{e^{2} \omega^{3}}{4 \pi^{2} c^{3}} \frac{Z l}{n} R^{2} \exp \left[\frac{-2 x_{0}}{\lambda_{e}}\right] .
$$

Alternatively, in terms of emission angle $\theta$ :

$$
\left(\frac{d I}{d \Omega}\right)_{1}=2 \pi e^{2} \frac{Z}{l^{2}} \frac{n^{2} \beta^{3}}{(1-\beta \cos \theta)^{3}} R^{2} \exp \left[-\frac{2 x_{0}}{\lambda_{e}}\right] .
$$

It is precisely this factor $N$ that enhances the SPR compared to, say, diffraction radiation from a single aperture. This description has received some experimental support, typically at energies of a few $\mathrm{MeV}$ [13].

\section{B. Many electrons, coherence}

The above are applicable to the idealized case of one electron at a height $x_{0}$ above the grating. For a real beam, we assume that the distributions of the particles above and across the grating ( $x$ and $y$ directions, respectively) are Gaussian but the distribution of the particles in the direction of beam propagation ( $z$ axis) is unknown. The contributions of $N_{e}$ electrons will then add together according to

$$
\left(\frac{d I}{d \Omega}\right)_{N_{e}}=\left(\frac{d I}{d \Omega}\right)_{1}\left(N_{e} S_{\mathrm{inc}}+N_{e}^{2} S_{\mathrm{coh}}\right),
$$

where $S_{\text {inc }}$ is defined as

$$
S_{\mathrm{inc}}=\frac{1}{\sqrt{2 \pi} \sigma_{x}} \int_{0}^{\infty} e^{-2 x / \lambda_{e}} e^{-\left[\left(x-x_{0}\right)^{2} / 2 \sigma_{x}^{2}\right]} d x .
$$

For an uncorrelated distribution, the "coherent" integral $S_{\text {coh }}$ in (4) is defined as

$$
\begin{aligned}
S_{\mathrm{coh}}= & \left|\frac{1}{\sqrt{2 \pi} \sigma_{x}} \int_{0}^{\infty} e^{-x / \lambda_{e}} e^{-\left[\left(x-x_{0}\right)^{2} / 2 \sigma_{x}^{2}\right]} d x\right|^{2} \\
& \times\left|\frac{1}{\sqrt{2 \pi} \sigma_{y}} \int_{-\infty}^{\infty} e^{-i k_{y} y} e^{-\left[\left(y-y_{0}\right)^{2} / 2 \sigma_{y}^{2}\right]} d y\right|^{2} \\
& \times\left|\int_{-\infty}^{\infty} e^{-i \omega t} f(t) d t\right|^{2} .
\end{aligned}
$$

The quantity $f(t)$ that appears in the above expression is the, as yet unspecified, distribution of the particles in the time domain. If the coherent integral $S_{\text {coh }}$ is very small, the second term in the parentheses of Eq. (4) is insignificant. This is the case of continuous beams, when the contributions of the various electrons add in an incoherent way and the radiated intensity is just $N_{e} S_{\text {inc }}$ times that of a single electron. For such a beam it is probably more convenient to use an expression for the radiated power $P$ (rather than intensity) in terms of the beam current $I$ :

$$
\frac{d P}{d \Omega}=2 \pi e I \frac{Z}{l^{2}} \frac{n^{2} \beta^{3}}{(1-\beta \cos \theta)^{3}} R^{2} S_{\mathrm{inc}} .
$$

For bunched beams whose bunch length is comparable to or shorter than the wavelength of the radiation, $S_{\text {coh }}$ will dominate and there is "coherent enhancement" of the emitted radiation by a factor approaching $N_{e}$. This is critically dependent on the longitudinal (time) profile of the bunch.

We have considered a number of plausible time profiles $f(t)$ and have evaluated the coherent integral in each case: Gaussian, triangular, exponential, parabolic, and cosinelike. Although the details of the calculation are omitted here, the results are discussed and compared to the experiment in Sec. IV C.

\section{EXPERIMENTAL ARRANGEMENT}

\section{A. Beam parameters and grating}

The experiments were carried out at the ENEA FEL Facility at Frascati [14], using the electron beam produced by the Microtron accelerator. The machine is capable of 
producing beams at discreet energies, starting at $1.8 \mathrm{MeV}$ and extending to $5 \mathrm{MeV}$, in steps of $0.8 \mathrm{MeV}$. All the experiments reported here were carried out at $1.8 \mathrm{MeV}$ $(\gamma=4.52)$. The pulse structure of the accelerator consists of approximately 15 ps long bunches, spaced 333 ps apart. The pulse train duration is $5 \mu \mathrm{s}$ and the average current in the pulse train was, typically, $200 \mathrm{~mA}$; hence, each bunch contains about $4.2 \times 10^{8}$ electrons. The normalized emittance of the beam is approximately $50 \pi \mathrm{mm} \mathrm{mrad}$ and the repetition rate about $1 \mathrm{~Hz}$.

The grating was mounted in the multipurpose interaction chamber of the facility, designed to host undulators, Cherenkov, and grating FELs. It had a profile consisting of two facets, one at a blaze angle of $14^{\circ}$ relative to the beam direction and the other vertical to the first. Its period was $2.5 \mathrm{~mm}$, its overall length $100 \mathrm{~mm}$, and its width $20 \mathrm{~mm}$. It was mounted on an insulated support so that any current intercepted could be measured.

\section{B. The optical system}

The optical system collects the emitted radiation and transports it to the detector. It has been designed for easy variation of the angle of observation (i.e., wavelength) and for efficient collection of light over a wide range of emission angles. It is shown in schematic form in Fig. 1.

It consists of three gold-plated copper mirrors, held together in a rigid frame and rotating as a unit around a $y$ axis, through the center of the grating. The range of angles accessible by the unit lies between $30^{\circ}$ and $170^{\circ}$. The system is also capable of collecting light at azimuthal angles $\phi$ up to $30^{\circ}$, but all the measurements reported here were carried out at $\phi=0^{\circ}$, with respect to the normal to the grating surface. The maximum solid angle of the system is limited by the last mirror and is equal to $0.05 \mathrm{sr}$. At a given position of the 3-mirror frame, there is a range of emission angles $\theta$ that can be collected by the mirrors and transmitted, typically about $\pm 14^{\circ}$. As the 3-mirror assembly rotates, the effective length of the grating and the range of emission angles accepted by the system change. For example, when the frame is positioned at $90^{\circ}$, values

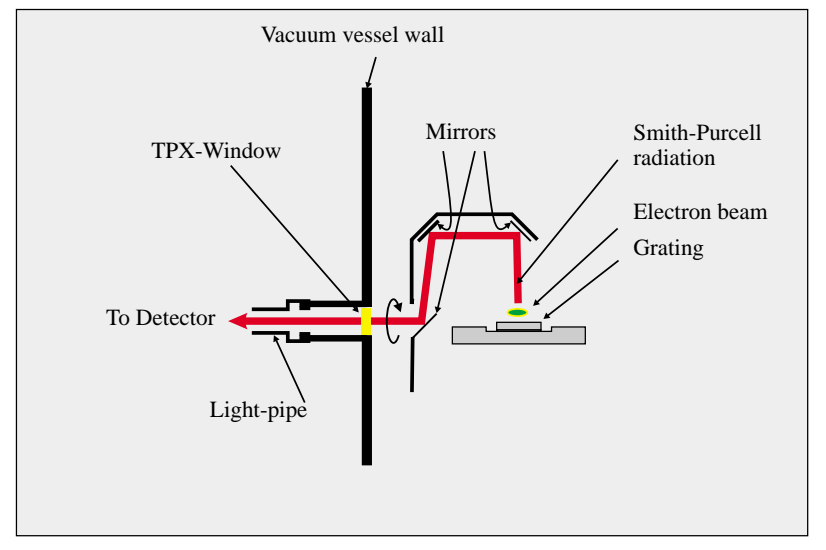

FIG. 1. (Color) Schematic of light-collection system. of $\theta$ between $76^{\circ}$ and $104^{\circ}$ are accepted and the effective grating length is $40 \mathrm{~mm}$; this rises to about $70 \mathrm{~mm}$ at $40^{\circ}$. Radiation is extracted from the side of the vacuum chamber and, by means of a copper light pipe, is taken to the detector. The light pipe is separated from the vacuum chamber and from the outside world by TPX windows and can be evacuated in order to reduce absorption by the atmosphere; this has not been necessary for the wavelengths reported here. The transmission efficiency of the optical system was measured by placing a "hot" $\left(\cong 600{ }^{\circ} \mathrm{C}\right)$ source first in the position of the grating and then in front of the detector and comparing the two signals. The efficiency is $0.65 \pm 10 \%$.

\section{The detector}

An InSb electron bolometer, cooled to liquid helium temperature, was used to detect the radiation emerging from the window at the end of the light pipe. The detector was placed behind a screen of lead blocks, in order to reduce the bremsstrahlung background, and was calibrated using an optically pumped far-infrared laser and millimeter wave sources of known power. Its responsivity is $1500 \mathrm{~V} / \mathrm{W}$ at $\lambda=0.6 \mathrm{~mm}$, rising to $2250 \mathrm{~V} / \mathrm{W}$ at about $\lambda=2 \mathrm{~mm}$ and is approximately flat thereafter. Its response time is less than $0.5 \mu \mathrm{s}$. To avoid saturation of the detector, calibrated mesh filters were employed to attenuate the signal. These had the further property of strongly attenuating unwanted longer wavelength radiation, which was also transmitted by the light pipe and detected by the bolometer. A Fabry-Perot interferometer was inserted between the end of the light pipe and the detector in order to determine the wavelength of the SPR.

\section{RESULTS AND DISCUSSION}

\section{A. Fabry-Perot interferometer scans}

The experimental procedure was as follows: using the quadrupole lenses and beam deflectors, the electron beam was positioned about $1 \mathrm{~mm}$ above the grating. Typically, the beam size at the center of the grating was $2 \times 4 \mathrm{~mm}$, in the $x$ and $y$ directions, respectively. The 3-mirror assembly was rotated to a given angle $\theta$ and the Fabry-Perot was used to scan the wavelengths. The Fabry-Perot mirrors were wire meshes, which for most of the measurements had a density of 200 lines/in. The "finesse" (or resolution) of the instrument depends on the flatness and the reflectivity of the two mirrors. In our case it is estimated that the finesse was $\cong 50$. Therefore, at a wavelength of $2.5 \mathrm{~mm}$ the resolution is $50 \mu \mathrm{m}$, approximately. Typical scans, taken at $\theta=50^{\circ}, \theta=90^{\circ}$, and $\theta=120^{\circ}$, are shown in Figs. 2(a)-2(c), respectively. The measurements were limited to angles between $40^{\circ}$ and $120^{\circ}$ because of the strong background signal, probably from the Microtron itself, in the wavelength region of a few $\mathrm{mm}$; at low mirror angles, when the coherence effects are weak, 

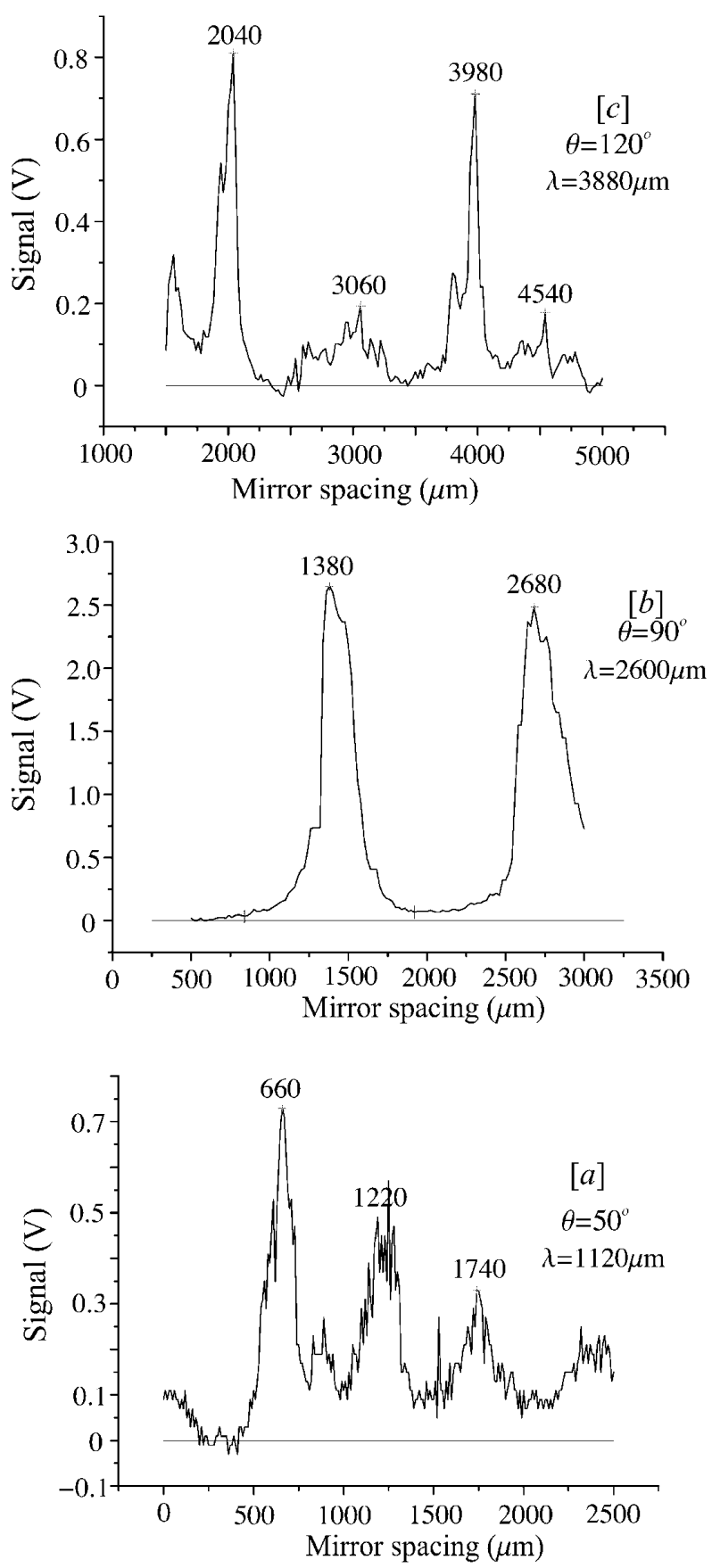

FIG. 2. Three Fabry-Perot scans taken with the mirrors at (a) $50^{\circ}$, (b) $90^{\circ}$, and (c) $120^{\circ}$.

the background masks the SP signal, while at angles over $120^{\circ}$ the SPR wavelength becomes indistinguishable from that of the background. This is a problem that will be dealt with in future experiments.

Since there is an inevitable "zero position" uncertainty for the two mirrors of the Fabry-Perot, the peak wavelength can be derived from Fig. 2, not from the nominal position of the first peak but by equating the difference between two successive peaks to $\lambda / 2$. Thus in Fig. 2(a) the peak wavelength collected at $\theta=50^{\circ}$ is $1.12 \mathrm{~mm}$; for $\theta=90^{\circ}$

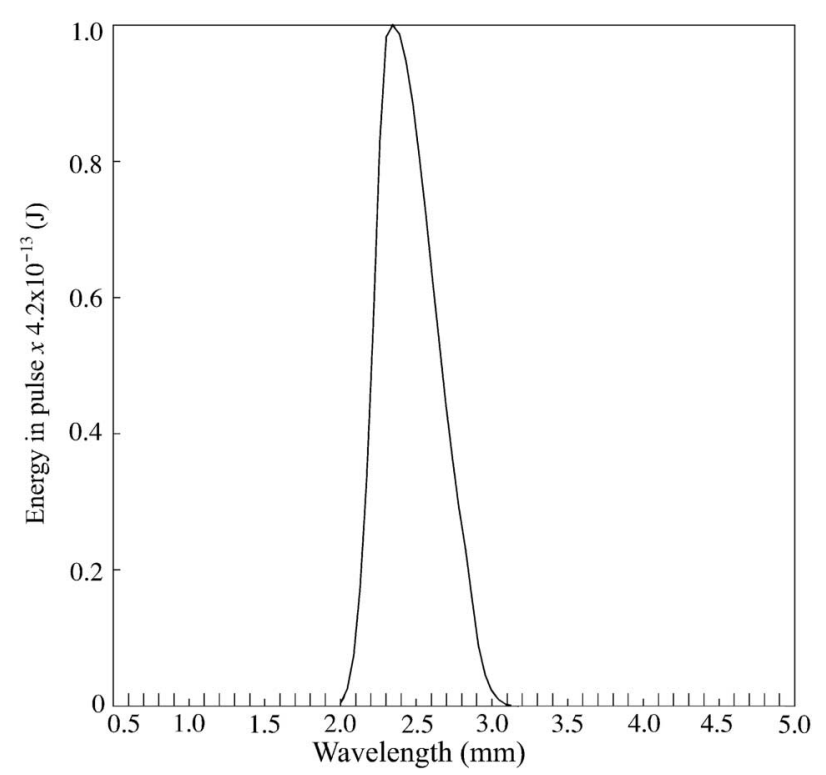

FIG. 3. Calculated energy versus wavelength, with the mirror assembly at $90^{\circ}$. The pulse shape is assumed to be triangular with an asymmetry factor $\epsilon=1.1$.

and $\theta=120^{\circ}$ the corresponding figures are $2.6 \mathrm{~mm}$ and $3.88 \mathrm{~mm}$, respectively. Note the difference in the calibration of the axes in the three figures. At an angle of $120^{\circ}$ the SPR has a wavelength that is sufficiently long $(3.88 \mathrm{~mm})$ for its second harmonic $(1.94 \mathrm{~mm})$ to be coherently enhanced. This is evident in Fig. 2(c), where the spacing between the peaks 2040, 3060, and 3980 corresponds (approximately) to half the wavelength of the second harmonic. The area under one of the scan peaks must contain all the wavelengths collected by the optical system at a given angle $\theta$ of the mirrors and, in fact, the two main peaks at 2040 and 3980 of Fig. 2(c) must also contain a percentage of the second harmonic (see following section). Figure 3 shows the calculated energy versus wavelength, with the mirror assembly set at $\theta=90^{\circ}$, taking into account the geometry of the optical system, the solid angle, and the effective length of the grating. Although this calculation is based on an assumed triangular time profile of the pulse, it is typical for other profiles as well and indicates a full width at half maximum of about $470 \mu \mathrm{m}$. The measured FWHM from Fig. 2(b) is $2 \times 220 \mu \mathrm{m}$, in reasonable agreement with the theoretical expectation.

\section{B. Analysis}

The starting point of the analysis is the observation that, provided that the instrumental resolution is much smaller than the range of wavelengths accepted by the optical system, then the area under the first peak of the Fabry-Perot scan is proportional to the power collected by the mirror assembly. For every mirror angle, repeated scans were taken and an average value for the area under the peak was obtained. This was then normalized to the value measured 
at $\theta=90^{\circ}$. Since the responsivity of the detector in the wavelength range covered is not constant, an appropriate correction factor was applied to the shorter wavelengths, i.e., the smaller mirror angles. Theoretical estimates indicate that the 2 nd harmonic content is less than $10 \%$ for emission angles smaller than $90^{\circ}$ and can be absorbed in the analysis errors, which are discussed in Sec. IV D. For emission angles above $100^{\circ}$, approximately, there is a significant contribution from the 2 nd harmonic, which contributes about $15 \%$ of the signal at $100^{\circ}$ and $35 \%$ at $120^{\circ}$. This fraction was subtracted from the measured values above $90^{\circ}$. Finally, all the values were divided by the transmission efficiency of the light pipe (0.65) in order to derive the power at the entrance of the light pipe.

The final step in the analysis is to assign an absolute power to the normalizing position, i.e., at $90^{\circ}$. This was done in three ways: (i) The Fabry-Perot was replaced by a Czerny-Turner grating monochromator of known efficiency at the wavelength corresponding to an angle of $90^{\circ}$; the value obtained was $320 \mathrm{~mW}$. (ii) Using suitable mesh filters, as discussed in Sec. IIIC, and the calibrated responsivity of the detector, a figure of $320 \mathrm{~mW}$ was also obtained. (iii) By replacing the InSb detector with a pyroelectric detector of known responsivity, placed at the exit of the light pipe and without the use of any spectrometer or filters, a figure of $400 \mathrm{~mW}$ was derived. In view of the strong background at long wavelengths and the assumption that the pyroelectric detector must have some sensitivity to long wavelengths, we have assigned $320 \mathrm{~mW}$ as the reference power at $90^{\circ}$.

\section{Discussion}

The first observation to make is that these power levels are significant and, at $90^{\circ}$, about 6 orders of magnitude higher than what would be expected from incoherent emission alone. This can be seen clearly from the two plots in Fig. 4, which show the expected levels of infrared radiation for the cases of spontaneous incoherent and coherent emission; the measured power levels extend up to a few hundred $\mathrm{mW}$ and are a strong indication of coherent enhancement.

The determination of the bunch profile is done by comparison of the measured angular distribution of the emitted coherent power with what would be expected from various possible temporal profiles. Assuming, as a simplifying approximation, that there is uniform particle density along the length of the pulse, this is a three-parameter fit, namely pulse shape, pulse length, and pulse asymmetry relative to the peak particle at time $t=0$. The latter is characterized by the factor $\epsilon$ by which the length of the pulse for $t<0$ differs from that with $t>0$; hence, $\epsilon=1$ represents a profile symmetric with respect to $t=0$. The bunch length is critical but it is constrained within the known limits of this bunch, $15 \pm 1$ ps. The data points are shown in Fig. 5. Also shown in the same figure are three theoreti-

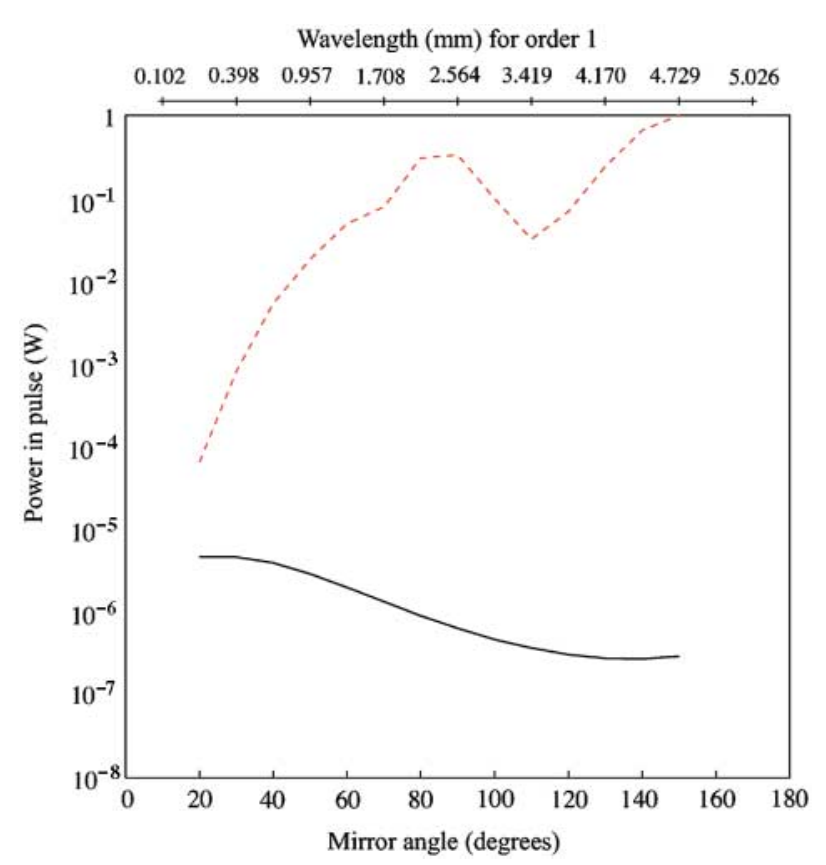

FIG. 4. (Color) Expected incoherent (solid line) and coherent (dashed line) output power levels for an assumed triangular pulse with $\epsilon=1.1$ (see text for details).

cally predicted angular distributions, marked as 1,2 , and 3 , corresponding to bunches with Gaussian, triangular, and exponential profiles, respectively. The Gaussian and exponential profiles have been assumed to be symmetric but the triangular one has a small degree of asymmetry. The

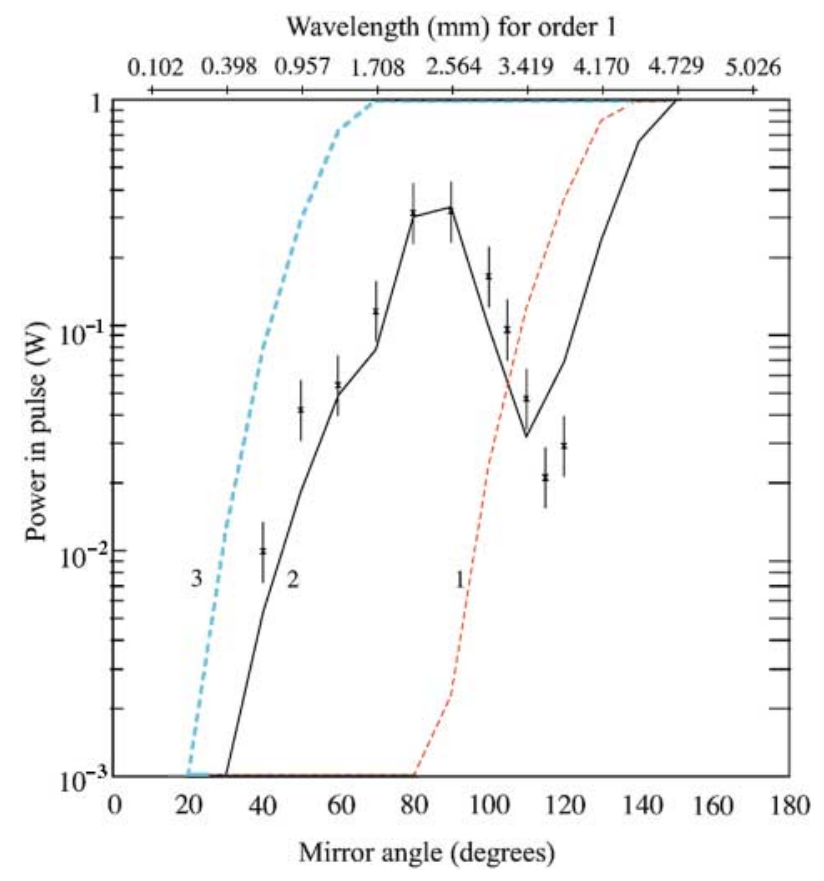

FIG. 5. (Color) Experimental data points, fitted with various longitudinal profiles, all containing $85 \%$ of the bunch particles inside 14 ps. 1: Gaussian; 2: triangular with asymmetry factor $\epsilon=1.1$; and 3: exponential. 
derivation of these curves starts with an estimate of the differential power output $(\mathrm{W} / \mathrm{sr} / \mathrm{cm})$, in steps of $1^{\circ}$, including the pulse-dependent coherence effects of Sec. II B. The geometric properties of the light-collecting system are then taken into account. As mentioned in Sec. III B, for each position of the mirror assembly a range of emission angles of about $28^{\circ}$ is accepted by the system, each with its own solid angle (typically $0.014 \mathrm{sr}$ ) and effective grating length. These are calculated, multiplied by the differential power, and added together in order to give the total power, in $\mathrm{W}$, entering the light pipe at that particular position of the mirror assembly. This can then be compared directly to the measured values. Plausible values for the asymmetry factor $\epsilon$ have been explored. All three curves of Fig. 5 contain about $85 \%$ of the bunch particles within 14 ps. The experimental points are well below the predictions of an exponential shape and above those of a Gaussian. On the other hand, a triangular profile with an asymmetry factor $\epsilon=1.1$ seems to be in reasonable agreement with the experiment. An interesting feature of this profile, and indeed of all profiles with sharp "kinks," is the appearance of interference minima, in this case at about $110^{\circ}$. These can be understood, in qualitative terms, as arising from the interference of some of the many harmonics that are bound to exist in the Fourier spectrum of a shape that contains kinks. The anticipated decrease in power above $90^{\circ}$ is reproduced well by the data but will have to be established more firmly by extending the range of measurement up to about $140^{\circ}$. The degree of asymmetry $\epsilon$ primarily governs the depth of the interference minima. Two other bunch shapes, parabolic and cosinelike (not shown in Fig. 5), have been tried; although there is little difference from the triangular one at mirror angles less than about $70^{\circ}$, they predict increasing emission up to $120^{\circ}$ and are, thus, in disagreement with the data. It is reasonable to exclude exponential, Gaussian, parabolic, and cosinelike shapes and to conclude that the shape of the bunch, under the operating conditions prevailing at the time, is approximately triangular. A wider range of angular distribution measurements, under different operating conditions, is required before a definitive statement can be made on the bunch shape of the Frascati Microtron. This work is in progress.

\section{Error estimate}

There are systematic and statistical errors in this experiment and the former are the dominant ones. The energy calibration at $90^{\circ}$, which was given as $320 \mathrm{~mW}$, has an estimated systematic error of $\pm 20 \%$ and the transmission efficiency from the entrance of the light pipe to the detector is $0.65 \pm 10 \%$. Therefore, the overall systematic error is $\pm 22 \%$. Potentially, the most significant source of error is the possible change of the bunch and bunch-train shape during the course of an experimental run, due to changes in the rf system of the accelerator. Although this would have a significant effect on the measured power (see Fig. 5), it is impossible to quantify. However, care was taken to ensure that the bunch-train shape was kept constant during a run and, hence, this source of error can be discounted for the set of measurements presented in this paper. The current can be read to an accuracy of $\pm 5 \%$, which results in an error of $\pm 10 \%$ in the power estimate. Beam position fluctuations contribute about $\pm 8 \%$, assuming the vertical beam position above the grating varies by $\pm 0.25 \mathrm{~mm}$. The error in the calculation of the area under a peak in the Fabry-Perot scan is estimated at about $\pm 20 \%$. This figure includes errors in the measurement of the area, the existence of a small contribution from the 2nd harmonic, and reproducibility errors in aligning the two mirrors of the Fabry-Perot interferometer. Combining all the above, we arrive at a total error of $\pm 32 \%$. The appropriate error bars have been placed on the data points of Fig. 5. Finally, the measurement of the mirror angle has an estimated error of $\pm 2.5^{\circ}$.

\section{SUMMARY AND CONCLUSIONS}

We have measured the absolute power levels of coherent Smith-Purcell radiation produced by the interaction of a $1.8 \mathrm{MeV}$ electron beam and a blazed grating with a period of $2.5 \mathrm{~mm}$ and blaze angle of $14^{\circ}$. The emission angle region covered was between $40^{\circ}$ and $120^{\circ}$, relative to the beam direction, corresponding to wavelengths in the range $0.65-3.88 \mathrm{~mm}$. The measured power levels varied from about $10 \mathrm{~mW}$ at $\theta=40^{\circ}$ up to $320 \mathrm{~mW}$ at $\theta=90^{\circ}$ and are in reasonable agreement with the predictions of a theoretical treatment of the emission process based on currents induced on the grating surface.

We have also used coherent Smith-Purcell radiation to determine the longitudinal profile of the Frascati Microtron bunch. One of the advantages of this radiative process is that it allows for easy collection of almost all of the emitted wavelengths by simple scanning of the angle of observation. In the present set of measurements we have been limited, for experimental reasons, to the above wavelength range. Nevertheless, this is the range where coherence effects start to dominate and, hence, there are significant differences in the power distributions produced by various bunch profiles. A comparison of the measured power levels with a number of assumed bunch profiles for the Frascati Microtron bunches (15 ps long) excludes the possibilities of a Gaussian or an exponential shape; it is, however, consistent with a nearly triangular shape, with a slight asymmetry relative to the peak particle.

Of particular interest is the applicability of SPR to bunch-shape diagnostics for $\mathrm{GeV}$ beams with ps or sub-ps bunch lengths. To the best of our knowledge, no experimental work has been done in that regime. Hence, we take as a reference point for the discussion that follows the case of a $1 \mathrm{GeV}(\gamma=2000)$ beam, containing $10^{9}$ particles in a $0.5 \mathrm{ps}$ bunch. The first consideration is the intensity of the radiated power. The coupling of the 
beam to the grating is determined by the exponential term in (2). For intense, highly relativistic beams one would almost certainly want to keep the beam at least $5 \mathrm{~mm}$ away from the grating. This will tend to reduce the coupling but this reduction will be compensated by the increase in the evanescent wavelength, because of the very high $\gamma$. Therefore, good coupling can be maintained and the radiated power will increase. The direction of the emitted spontaneous radiation will tilt in the forward direction but the coherence effects will dominate at the longer wavelengths, i.e., at angles of observation away from the electron beam direction. The selection of the optimum wavelength region is important. As mentioned in the introduction, this is one of the attractive features of SPR since it allows a selection of wavelength region by a suitable choice of the grating period, in order to suit a particular application. It can be observed in Fig. 5 that as the wavelength of the emitted radiation becomes larger than the pulse length, a situation of full coherence is approached and the information about the particular bunch shape is lost. The region of interest, in this experiment, was the one between $30^{\circ}$ and $140^{\circ}$ where the bunch shape did affect the angular distribution; whereas above $140^{\circ}$ ( $4.5 \mathrm{~mm}$, approximately) the three curves of Fig. 5 merge. However, for a much shorter bunch, say $0.5 \mathrm{ps}(0.15 \mathrm{~mm})$, the $2.5 \mathrm{~mm}$ grating is unsuitable and a grating with a shorter period, probably $0.1 \mathrm{~mm}$, would be required. We have carried out some calculations using a grating with a period of $0.1 \mathrm{~mm}$ and a blaze angle of $14^{\circ}$. Neither of these two values is meant to be an optimum; they are simply a first attempt to estimate what is possible under the above assumptions. The results are shown in Fig. 6. The vertical axis is calibrated in Joules per steradian per $\mathrm{cm}$ of grating length. The three curves refer to an exponential-shaped pulse ( $0.5 \mathrm{ps}$ long) and to two triangular shapes of 0.5 and $0.45 \mathrm{ps}$, respectively; each pulse is assumed to contain $85 \%$ of the particles and to have a slight degree of asymmetry $(\epsilon=1.1)$. The beam has been assumed to be $5 \mathrm{~mm}$ above the grating surface and to have a size of $0.1 \times 0.2 \mathrm{~mm}$.

As in the low energy case, the distinction between an exponential shape and a triangular one is clear. As far as the achievable "resolution" is concerned, the answer will depend to a great extent on the errors in the measurement. For the experiment reported here, and in spite of the considerable experimental errors, it was possible to distinguish between pulse lengths of 14 and 15 ps, i.e., of the order of $10 \%$. In the high energy, short pulse length case of Fig. 6 the resolution between 0.5 and 0.45 ps should also be possible at emission angles in the range $60^{\circ}$ to $160^{\circ}$, approximately. In this context, it also worth noting that a working device for longitudinal profile determination would not employ rotating mirrors for the collection of the various wavelengths. A better system might consist of an array of infrared detectors, positioned within the relevant range of emission angles, that will allow the measurement

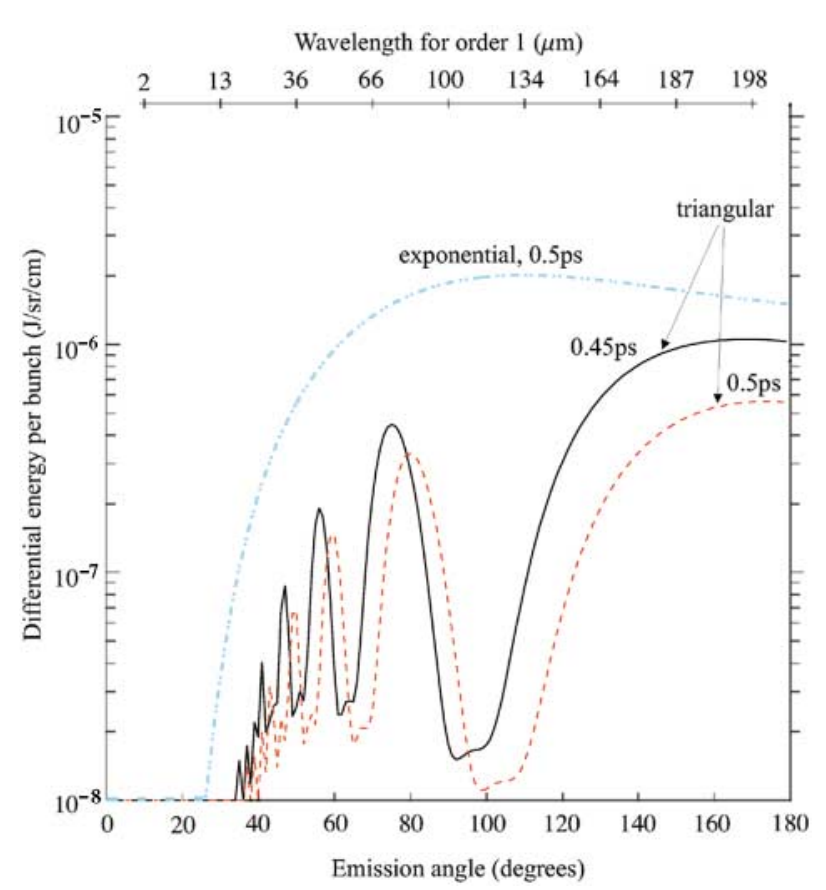

FIG. 6. (Color) Angular distribution of the radiated differential energy, for various longitudinal pulse shapes (see text for details).

of the angular distribution in a "single shot." Finally we note that as an alternative to gratings with short periods, it may be possible to use longer period gratings and a higher harmonic of the wavelength, thus taking advantage of the non-negligible power radiated in higher orders. For example, for the triangular profiles of Fig. 6 it is estimated that the 2 nd harmonic would have up to $20 \%$ of the peak energy of the 1st and the 3rd harmonic about $4 \%$.

We conclude that coherent SPR offers interesting possibilities as a sensitive, noninvasive tool for the determination of the shape of short (ps and sub-ps) bunches of relativistic electrons and for the measurement of longitudinal emittance.

\section{ACKNOWLEDGMENTS}

Two of the authors (G.D. and M. F. K.) would like to acknowledge the financial support of the Royal Society. J.H.B. and H. L. A. gratefully acknowledge the support of USARO Grant No. DAAD 19-99-1-0067 and NSF Grant No. ECS 0070491. We are grateful to Bill Linford for the expert construction of the light-collecting system, to Tony Jordan for the manufacture of the gratings, and to Antonio Fastelli for technical assistance at the ENEA FEL facility.

[1] Y. Shibata, T. Takahashi, T. Kanai, K. Ishi, M. Ikezawa, J. Ohkuma, S. Okuda, and T. Okada, Phys. Rev. E 50, 1479 (1994).

[2] J. Rosenzweig, G. Travish, and A. Tremain, Nucl. Instrum. Methods Phys. Res., Sect. A 365, 255 (1995). 
[3] H. Lihn, P. Kung, C. Settakorn, H. Wiedemann, and D. Bocek, Phys. Rev. E 53, 6413 (1996).

[4] R. B. Fiorito, D.W. Rule, and W. D. Kimura, in Advanced Accelerator Concepts: Eighth Workshop, AIP Conf. Proc. No. 472 (AIP, New York, 1999), p. 725.

[5] J. Urakawa, H. Hayano, K. Kubo, S. Kuroda, N. Terunuma, M. Kuriki, T. Okugi, T. Naito, S. Araki, A. Potylitsyn, G. Naumenko, P. Karataev, N. Potylitsyna, I. Vnukov, T. Hirose, R. Hamatsu, T. Muto, M. Ikezawa, and Y. Shibata, Nucl. Instrum. Methods Phys. Res., Sect. A 472, 309 (2001).

[6] A. H. Lumpkin, N. S. Sereno, and D. W. Rule, Nucl. Instrum. Methods Phys. Res., Sect. A 475, 470 (2001).

[7] B. Feng, M. Oyamada, F. Hinode, S. Sato, Y. Shibata, and M. Ikezawa, Nucl. Instrum. Methods Phys. Res., Sect. A 475, 492 (2001).
[8] X. Yan, A. M. MacLeod, W. A. Gillespie, G. M. H. Knippels, D. Oepts, A. F. G. van der Meer, and W. Seidel, Phys. Rev. Lett. 85, 3404 (2000).

[9] G. Doucas, M. F. Kimmitt, J. H. Brownell, S. R. Trotz, and J.E. Walsh, Nucl. Instrum. Methods Phys. Res., Sect. A 474, 10 (2001).

[10] S. J. Smith and E. M. Purcell, Phys. Rev. 92, 1069 (1953).

[11] J.H. Brownell, J.E. Walsh, and G. Doucas, Phys. Rev. E 57, 1075 (1998).

[12] S. R. Trotz, J.H. Brownell, J.E. Walsh, and G. Doucas, Phys. Rev. E 61, 7057 (2000).

[13] J. H. Brownell, G. Doucas, M. F. Kimmitt, J. H. Mulvey, M. Omori, and J. E. Walsh, J. Phys. D 30, 2478 (1997).

[14] G. P. Gallerano, A. Doria, E. Giovenale, and A. Renieri, J. Infrared Phys. Technol. 40, 161 (1999). 